\title{
Statistical study of undulator radiated power by a classical detection system in the mm-wave regime
}

\author{
A. Eliran and A. Gover \\ Department of Physical Electronics, Faculty of Engineering, Tel Aviv University, Tel Aviv, Israel \\ Y. Pinhasi, A. Yahalom, and Y. Lurie \\ Department of Electrical and Electronic Engineering, Ariel University Center of Samaria, Ariel, Israel \\ G. Pinhasi \\ Department of Chemical Engineering, Ariel University Center of Samaria, Ariel, Israel
}

(Received 27 February 2008; published 5 May 2009)

\begin{abstract}
The statistics of FEL spontaneous emission power detected with a detector integration time much larger than the slippage time has been measured in many previous works at high frequencies. In such cases the quantum (shot) noise generated in the detection process is dominant. We have measured spontaneous emission in the Israeli electrostatic accelerator FEL (EA-FEL) operating in the mm-wave lengths. In this regime the detector is based on a diode rectifier for which the detector quantum noise is negligible. The measurements were repeated numerous times in order to create a sample space with sufficient data enabling evaluation of the statistical features of the radiated power. The probability density function of the radiated power was found and its moments were calculated. The results of analytical and numerical models are compared to those obtained in experimental measurements.
\end{abstract}

DOI: 10.1103/PhysRevSTAB.12.050701

PACS numbers: 29.40.-n, 29.85.Fj, 29.20.Ba

\section{INTRODUCTION}

Electron devices such as microwave tubes and freeelectron lasers (FELs) utilize distributed interaction between an electron beam and an electromagnetic wave. Random electron distribution in the $e$-beam causes fluctuations in current density, identified as shot noise in the beam current. Electrons passing through a magnetic undulator emit radiation, which is called undulator synchrotron radiation [1]. The electromagnetic fields excited by each electron add incoherently, resulting in spontaneous emission having a certain power spectral density [2].

Saldin [3] derived an analytical proportionality relation between the radiation field and the beam current in the frequency domain, applicable for both spontaneous emission and self-amplified spontaneous emission (SASE) which is generated in the high gain regime. Saleh [4] made a distinction between the case of classical detection, as was used in this work, and the case of photon counting by a quantum detector. In the case of detection by use of a diode rectifier, the variance of the number of emitted photons $k$ is given by

$$
\sigma_{k}^{2}=\frac{\overline{k^{2}}}{M} \propto \sigma_{P_{T}}^{2}=\frac{\overline{P_{T}}}{M}
$$

and $k$ is described by the Gamma distribution where $M$ is the number of modes. $P_{T}$ is the detected power [Eq. (12)]. In the case of quantum detection, the variance is given by

$$
\sigma_{k}^{2}=\overline{k^{2}}-\bar{k}^{2}=\bar{k}+\bar{k}^{2}
$$

where the number of photons $k$ is distributed by the BoseEinstein or geometric probability density function. The above is for a single mode, while in the multimode case the radiation is described by the negative-binomial distribution, with variance

$$
\sigma_{k}^{2}=\overline{k^{2}}-\bar{k}^{2}=\bar{k}+\frac{\bar{k}^{2}}{M},
$$

where $M$ is the number of modes.

Comparison of (3) and (1) shows that in the case of photon counting the term $\bar{k}$ is added, which is the so-called quantum noise. In this case the quantum noise is a significant part of the total noise, whereas for the diode rectifier it is negligible.

Tanabe et al [5], at the Brookhaven National Laboratories, measured the negative-binomial distribution the number of photons spontaneously emitted by their FEL.

In this work we study the statistical distribution of the spontaneous radiation power measured in the $\mathrm{mm}$-wave regime for our electrostatic accelerator FEL (EA-FEL).

\section{SPONTANEOUS EMISSION}

The total spontaneous emission field is given by a summation of the field radiated by each electron entering the wiggler. The field contributions of different wave packets are independent random variables. Therefore, following the central limit theorem [6], for large $n$ the probability density function of the field is Gaussian with zero mean and variance $\sigma_{E}^{2}$. 
The power spectral density that results from the sum of electromagnetic fields excited by electrons passing through a magnetic undulator added incoherently is according to [2]

$$
\frac{d P_{\mathrm{sp}}}{d f}\left(L_{w}\right)=\tau_{\mathrm{sp}} P_{\mathrm{sp}}\left(L_{w}\right) \sin c^{2}\left(\frac{1}{2} \theta L_{w}\right),
$$

where $P_{\mathrm{sp}}\left(L_{w}\right)$ is the expected value of the total spontaneous emission power at the end of the undulator of length $L_{w}, \tau_{\mathrm{sp}}=\left|\frac{L_{w}}{v_{z}}-\frac{L_{w}}{v_{g}}\right|$ is the slippage time, and $\theta=\frac{\omega}{v_{z 0}}-$ $\left(k_{z q}+k_{w}\right)$ is the detuning parameter. In the above equations $v_{z 0}$ is the electron axial velocity, $v_{g}$ is the group velocity of the radiation wave packet, $k_{z}$ is the axial wave number, $k_{w}$ is the wiggler wave number, and $\omega$ is the angular frequency. The spontaneous emission null-tonull bandwidth is $\frac{2}{\tau_{\mathrm{sp}}} \simeq \frac{2 f_{0}}{N_{w}}$, in which $f_{0}$ corresponds to the central frequency for which the detuning is $\theta=0$ and $N_{w}$ is the number of periods in the FEL wiggler.

In an FEL utilizing a magnetostatic planar wiggler, the total average power of the spontaneous emission is given by [2]

$$
P_{\mathrm{sp}}\left(L_{w}\right)=\frac{1}{8} A_{J J}^{2} \frac{e I_{0}}{\tau_{\mathrm{sp}}}\left(\frac{a_{w}}{\gamma \beta_{z}}\right)^{2} \frac{Z_{01}}{A_{\mathrm{em}}} L_{w}^{2},
$$

where

$$
A_{J J}=J_{0}\left[\frac{a_{w}^{2}}{2\left(a_{w}^{2}+2\right)}\right]-J_{1}\left[\frac{a_{w}^{2}}{2\left(a_{w}^{2}+2\right)}\right] .
$$

In our case, since $a_{w} \ll 1$, we obtain that $A_{J J} \cong 1$.

The spontaneous emission average power is proportional to $e I_{0}$, where $I_{0}$ is the DC beam current and $e$ is the electron charge. The wiggler parameter is $a_{w}, \beta_{z}=\frac{v_{z 0}}{c}$ in which $c$ is the velocity of light in vacuum, and the relativistic Lorentz factor is defined as

$$
\gamma=\sqrt{\frac{1}{1-v^{2} / c^{2}}} .
$$

The mode effective area is given by

$$
A_{\mathrm{em}, 01}=\frac{a_{\mathrm{eff}} b_{\mathrm{eff}}}{2}=4.55 \times 10^{-5} \mathrm{~m}^{2}
$$

and the mode impedance is given by

$$
Z_{01}=Z_{0} \frac{k}{\sqrt{k^{2}-k_{c}^{2}}},
$$

where $k$ is the free space wave number,

$$
k=\frac{\omega}{c},
$$

$k_{c}$ is the cutoff wave number,

$$
k_{c}=\frac{2 \pi f_{c}}{c},
$$

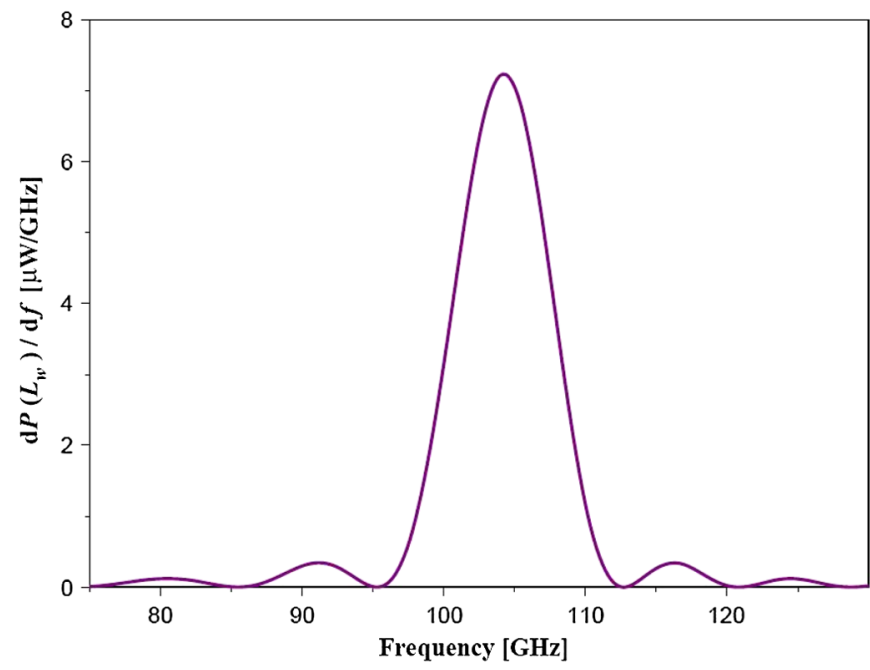

FIG. 1. Power spectral density of spontaneous emission for typical parameters.

$f_{c}$ is the cutoff frequency,

$$
f_{c} \approx c \frac{\pi}{b_{\mathrm{eff}}},
$$

and $b_{\text {eff }}$ is the smaller waveguide dimension (Table I).

In the low gain limit, the spontaneous emission power grows linearly with the interaction length $L_{W}$.

The spontaneous power spectral density calculated for the electrostatic accelerator FEL with the parameters given in Table I is described in Fig. 1. This EA-FEL is designed to operate in the mm-wave regime.

\section{A. Probability density of the radiated power}

The total spontaneous emission power averaged over a time interval $T$ is

$$
P_{T}=\frac{1}{2 Z_{0}} \frac{1}{T} \int_{0}^{T} E^{2}(t) d t .
$$

It is a random variable with mean $\bar{P}_{T}=P_{\mathrm{sp}}$ characterized statistically by the Gamma distribution $[3,4,6]$

$$
f_{P_{T}}\left(P_{T}\right)= \begin{cases}\left(\frac{M}{P_{\mathrm{sp}}}\right)^{M} \frac{P_{T}^{M-1} e^{-M\left(P_{T} / P_{\mathrm{sp}}\right)}}{\Gamma(M)} & P_{T} \geq 0 \\ 0 & P_{T}<0 .\end{cases}
$$

$\Gamma$ stands for the Gamma function. The parameter $M$ is the number of modes within the detection integration time. In this context we define modes as random wave packets of coherence time $\tau_{\mathrm{sp}}$ (not resonator modes!) and is given by (15) below.

It is the number of coherence lengths [3,7] within the integration interval $T$.

As $M \rightarrow 1$, the negative-exponential distribution is obtained. For very high values of $M$, this is the sum of a very large number of random variables, and, according to the central limit theorem, the distribution becomes Gaussian. 
TABLE I. Typical operation parameters of the Israeli EA-FEL.

\begin{tabular}{|c|c|}
\hline Magnetostatic planar wiggler & \\
\hline $\begin{array}{l}\text { Magnetic induction } \\
\text { Period } \\
\text { Number of periods }\end{array}$ & $\begin{array}{c}B_{w}=2 \mathrm{kG} \\
4.444 \mathrm{~cm} \\
20\end{array}$ \\
\hline \multicolumn{2}{|l|}{ Waveguide } \\
\hline $\begin{array}{l}\text { Parallel curved plated } \\
\text { Effective mode dimensions } \\
\text { Radiation mode }\end{array}$ & $\begin{array}{c}R=17.2 \mathrm{~mm}, b=10.7 \mathrm{~mm} \\
a_{\mathrm{eff}} \times b_{\mathrm{eff}}=1.01 \mathrm{~cm} \times 0.9005 \mathrm{~cm} \\
\mathrm{TE}_{01}\end{array}$ \\
\hline \multicolumn{2}{|l|}{ Accelerator } \\
\hline $\begin{array}{l}\text { Beam energy } \\
\text { Beam radius } \\
\text { Beam current }\end{array}$ & $\begin{array}{c}1.4 \mathrm{MeV} \\
r_{b}=2 \mathrm{~mm} \\
I_{0}=1 \mathrm{~A}\end{array}$ \\
\hline \multicolumn{2}{|l|}{ Spontaneous emission (theory) } \\
\hline $\begin{array}{l}\text { Slippage time } \\
\text { Theoretical spontaneous emission power generation } \\
\text { Power expected at the detector }\end{array}$ & $\begin{array}{c}\tau_{\mathrm{sp}}=\left|\frac{L_{w}}{v_{z}}-\frac{L_{w}}{v_{g}}\right|=0.112 \mathrm{~ns} \\
68 \mu \mathrm{W} \\
0.5 \mu \mathrm{W}\end{array}$ \\
\hline
\end{tabular}

The variance of the power is

$$
\sigma_{P_{T}}^{2}=\frac{P_{\mathrm{sp}}^{2}}{M} .
$$

\section{EXPERIMENTAL RESULTS}

In order to compare theoretical and numerical results with experiment, additional components of the measurement setup must be taken into account. Figure 2 illustrates the measurement setup. The detector is a GaAs diode detector. Its $-3 \mathrm{~dB}$ video bandwidth is $B=10 \mathrm{MHz}$. The integration interval's length is $T=1 / 2 \pi B=16 \mathrm{~ns}$. Since the round-trip time of the resonator is approximately $10 \mathrm{~ns}$, and since the radiation pulse of one electron is the sequence of fully correlated pulses of length $\tau_{\mathrm{sp}}$, delayed by $10 \mathrm{~ns}$ from each other, two side peaks of correlation function give contribution to the integral. Thus [6]

$$
M=\frac{T}{3 \tau_{\mathrm{sp}}} .
$$

Taking $\tau_{\mathrm{sp}}$ from Table I and using (15), we find $M=47$.

Figure 3 shows the model for the diode detector with its equivalent noise sources [8]. No bias voltage is applied. The spreading resistance, $R_{s}$, lies typically between $1 \Omega$ and $50 \Omega$. This is the Ohmic resistance associated with the semiconductor outside the junction and the contacts. The dynamic resistance of the junction, $R_{j}$, at zero bias is

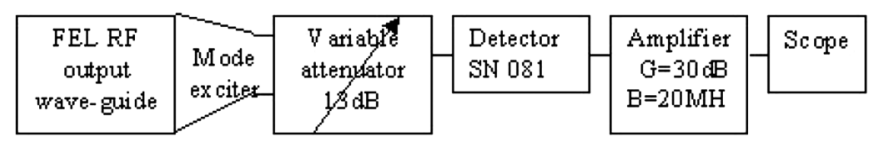

FIG. 2. Measurement setup.
$2.2 \mathrm{k} \Omega . C_{j}$ is the junction capacitance and its value is $30 \mathrm{fF}$. $R_{L}$, the load resistance, is the amplifier's input impedance. Its value is $20 \mathrm{k} \Omega$. Total detection system responsivity is $R=v_{\text {out }} / P_{\text {rf }}=1 \mu \mathrm{V} / \mathrm{nW}$.

We measured the power of each of one-hundred subsequent pulses of spontaneous emission, trying to maintain FEL conditions as stable as possible from one pulse to the next. The power at $3 \mu \mathrm{s}$ after the beginning of the pulse, when the power has already reached its peak, was taken as the random variable. Figure 4 shows a few examples of the pulses captured. The average power obtained was $P_{\mathrm{sp}}=$ $484 \mathrm{nW}$, and the standard deviation was $\sigma_{P_{T}}=77 \mathrm{nW}$. The power measured at the detector is substantially lower than the theoretical value (see Table I), which was calculated from Eq. (5) for the power generation inside the

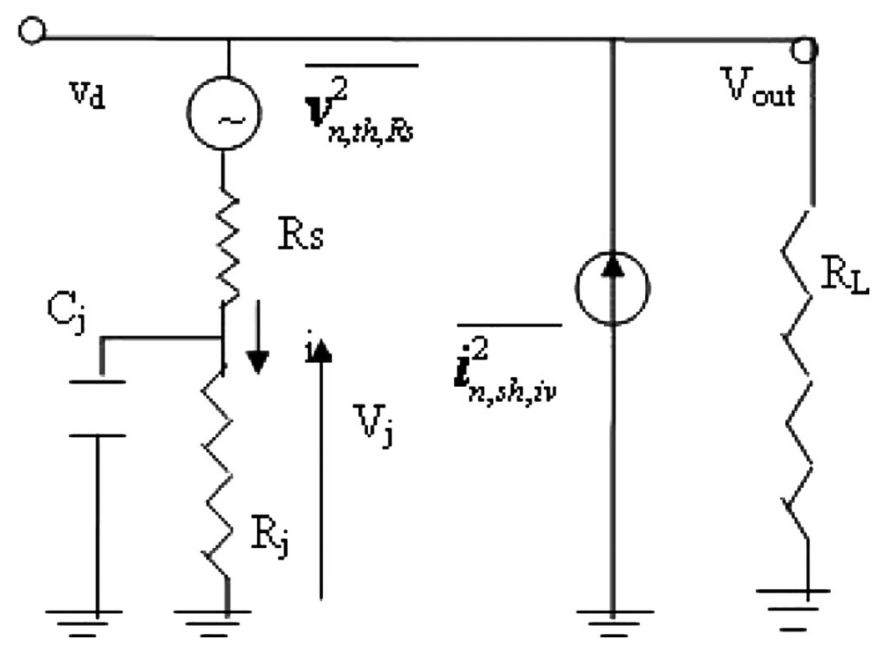

FIG. 3. Diode detector with equivalent noise sources. 

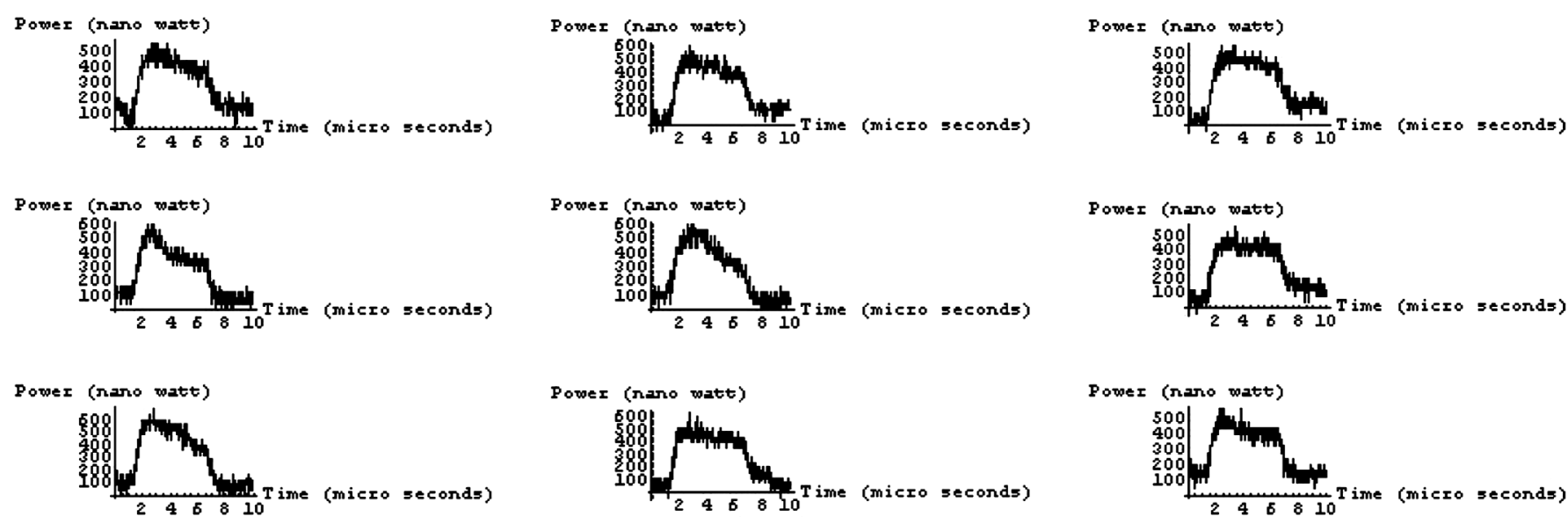

FIG. 4. A few of the pulse signals.

cavity due to the cavity out-coupling coefficient $1-R=$ 0.07 and substantial transmission loss of the waveguide transmission line.

Theoretically, a Gamma distribution is expected for the detected power [Eq. (13)], with the parameter $M$ being the number of modes [Eq. (15)].

We need now to eliminate the effect of the detector shot noise from the variance of the measured distribution. The detector output voltage is the sum of two additive components:

$$
\begin{gathered}
V_{\text {field }}=A_{v} \mathcal{R} P_{T} \\
V_{n, \text { out }}=\sqrt{\Delta v_{j, R_{s}}^{2}} F A_{v},
\end{gathered}
$$

where $v_{\text {field }}$ is the output voltage of the amplifier at the detector output, corresponding to the radiated power, $\mathcal{R}$ is the responsivity of the detector, $A_{v}$ is the voltage gain of the amplifier, and $V_{n \text {,out }}$ is the detector noise voltage at the amplifier output. $F$ is the noise figure.

The standard deviation of the measured detector noise power was $\sigma_{n}=\sqrt{\frac{\overline{v_{n}^{2}}}{\mathcal{R}}}=45 \mathrm{nW}$. The standard deviation of the measured data distribution (blue curve in Fig. 5) is $\sigma_{P_{T}}=77 \mathrm{nW}$. Since the detector noise and the radiation noise are statistically independent, we estimate the variance of the net radiation power distribution as the difference of the variances of the two distributions:

$$
\sigma_{\mathrm{sp}}=\sqrt{\sigma_{P T}^{2}-\overline{\sigma_{n}^{2}}}=63 \mathrm{nW} .
$$

This estimate should be compared to the calculated theoretical standard deviation that is calculated from (14) with the measured $P_{\mathrm{sp}}=484 \mathrm{nW}$ and the calculated $M=47$ [Eq. (15)].

The theoretical standard deviation that should be expected for this value of $M$ by (14) and (15) is

$$
\sigma_{P_{T}}=\frac{P_{\mathrm{sp}}}{\sqrt{M}}=70.6 \mathrm{nW}
$$

Figure 5 displays the following distributions: (i) The blue curve displays the distribution of the measured data. (ii) The dashed curve displays a normal distribution best fit to the experimental curve with $P_{\mathrm{sp}}=484 \mathrm{nW}$ and $\sigma_{P_{T}}=77 \mathrm{nW}$. (iii) The green curve displays the normal distribution of the net radiation power (detector noise subtracted) with $P_{\mathrm{sp}}=484 \mathrm{nW}$ and $\sigma_{\mathrm{sp}}=63 \mathrm{nW}$. (iv) The red curve displays the theoretical Gamma distribution for parameter $P_{\mathrm{sp}}=484 \mathrm{nW}$ and $M=47$, which correspond in reality (since $M \gg 1$ ) to a Gaussian distribution with $\sigma_{\mathrm{sp}}=70.6 \mathrm{nW}$.

Clearly, the theoretical distribution of the radiation power (red curve) is only slightly narrower than the power without subtraction of the detector noise. This widening

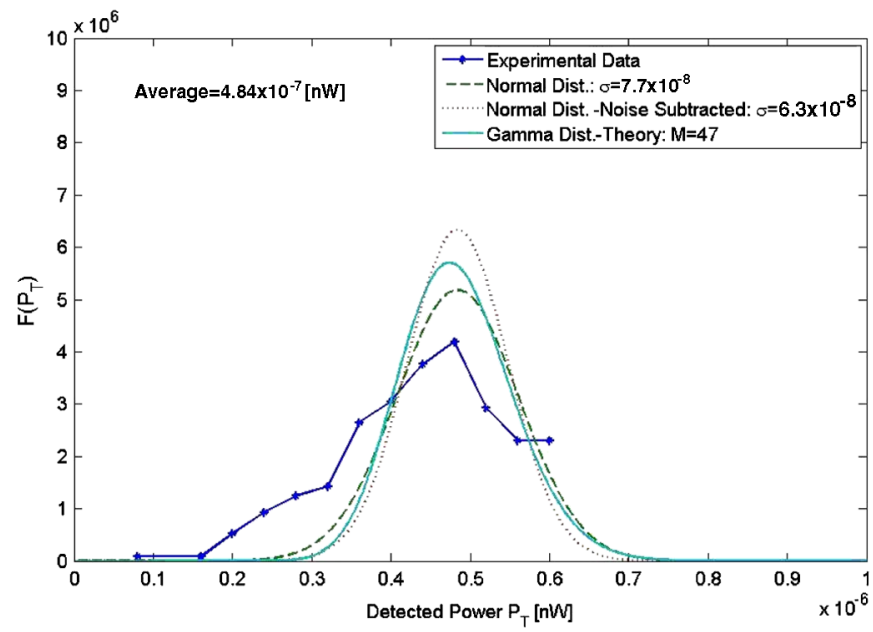

FIG. 5. (Color) Comparison of the experimental data to a Gamma distribution with the theoretical value $M=47$, the Gaussian approximation for the latter, with standard deviation $77 \mathrm{nW}$ and the normal distribution with standard deviation $63 \mathrm{nW}$ 
must be attributed to technical noise related to the instability of the accelerator parameters including instability of the accelerator terminal voltage, the $e$-beam current, and the $e$-beam deviated trajectories due to the electron-optics dispersion.

The results of the present experiment indicate that the measured statistical power distribution reflects the inherent features of the spontaneous emission originating from the $e$-beam shot noise. With proper stabilization of the electrostatic accelerator even better results may be achievable in the future.

The classical statistical optics that we implement in this article for the first time to FEL oscillators, predicts that the inherent statistical distribution is a Gamma distribution function. At the experimental parameters of the present setup, the number of modes correspond to $M>1$ and the distribution appears Gaussian (with or without the technical noise). Non-Gaussian distribution is definitely possible at the low frequency regime ( $\mathrm{mm}$ and $\mathrm{THz}$ waves). However, their observation requires faster detectors $(T \ll$ $1 \mathrm{~ns}$ ) or a longer wiggler.

Using the experimental values for the power mean and variance, $P_{\mathrm{sp}}=484 \mathrm{nW}$ and $\sigma_{P_{T}}=77 \mathrm{nW}$, we obtain by (14)

$$
M=\left(\frac{P_{\mathrm{sp}}}{\sigma_{P_{T}}}\right)^{2}=40
$$

which is close to the theoretical value 47 calculated above.

It should be noted that the noise voltage is also assumed Gaussian and, thus, the detected noise power also has the Gamma distribution. Finally, the sum of the two power components is Gamma distributed.

The Gamma distribution functions corresponding to $M=40$ and $M=47$ are shown on top of the experimental histogram in Fig. 5, as is the Gaussian approximation for the Gamma distribution with $M=40$. Since $M \gg 1$, the Gamma distribution tends to be Gaussian.

There is a reasonable fit between the width of the theoretically calculated distribution functions and the experimental histograms. The figure clearly shows that for the value $M=40$ the Gamma distribution function is closely approximated by the Gaussian function.

The theoretical calculated total generated power is according to Eq. (5) $P_{\mathrm{sp}}\left(L_{w}\right)=68 \mathrm{uW}$. The overall losses, including resonator round-trip Ohmic losses and outcoupling losses (together 80\%) and transport losses $(96 \%)$, are $21 \mathrm{~dB}$. Accordingly, the measured power at the detector was $0.5 \mathrm{uW}$.

\section{ACKNOWLEDGMENTS}

We thank Mr. Arie Eichenbaum for his valuable comments and suggestions.

[1] H. Motz, J. Appl. Phys. 22, 527 (1951).

[2] Y. Pinhasi and Yu. Lurie, Phys. Rev. E 65, 026501 (2002).

[3] E. L. Saldin, E. A. Schneidmiller, and M. V. Yurkov, The Physics of Free Electron Lasers (Springer, Berlin, 2000), p. 357.

[4] B. Saleh, Photoelectron Statistics (Springer, Berlin, 1978), Vol. 19, pp. 12, 19.

[5] T. Tanabe et al., Nucl. Instrum. Methods Phys. Res., Sect. A 304, 77 (1991).

[6] J. W. Goodman, Statistical Optics (John Wiley, New York, 1985), pp. 121, 238.

[7] Towards X-Ray Free Electron Lasers, edited by R. Bonifacio and W. A. Barletta, AIP Conf. Proc. No. 413 (American Institute of Physics, New York, 1997), p. 5.

[8] G. R. Nicoll, Proc. Inst. Elec. Eng. 101, 317 (1954). 